\title{
Unravelling the nature of HD 81032 - a new RS CVn Binary
}

\author{
J. C. Pandey ${ }^{1 *}$, K. P. Singh ${ }^{2 *}$, S. A. Drake ${ }^{3 *}$, and R. Sagar ${ }^{1 *}$ \\ 1 Aryabhatta Research Institute of Observational Sciences, Naini Tal - 263 129, India \\ 2 Tata Institute of Fundamental Research, Mumbai - 400 005, India \\ 3 USRA \& Code 662, NASA/Goddard Space Flight Center, Greenbelt, MD 20771, USA
}

\begin{abstract}
BVR photometric and quasi-simultaneous optical spectroscopic observations of the star HD 81032 have been carried out during the years 2000 - 2004. A photometric period of $18.802 \pm 0.07 \mathrm{~d}$ has been detected for this star. A large group of spots with a migration period of $7.43 \pm 0.07$ years is inferred from the first three years of the data. $\mathrm{H} \alpha$ and $\mathrm{Ca}$ II $\mathrm{H}$ and $\mathrm{K}$ emissions from the star indicate high chromospheric activity. The available photometry in the BVRIJHK bands is consistent with spectral type of K0 IV previously found for this star. We have also examined the spectral energy distribution of HD 81032 for the presence of an infrared colour excess using the 2MASS JHK and IRAS photometry, but found no significant excess in any band above the normal values expected for a star with this spectral type. We have also analyzed the X-ray emission properties of this star using data obtained by the ROSAT X-ray observatory during its All-Sky Survey phase. An X-ray flare of about 12 hours duration was detected during the two days of X-ray coverage obtained for this star. Its X-ray spectrum, while only containing 345 counts, is inconsistent with a single-temperature component solar-abundance coronal plasma model, but implies either the presence of two or more plasma components, non-solar abundances, or a combination of both of these properties. All of the above properties of HD 81032 suggest that it is a newly identified, evolved RS CVn binary.
\end{abstract}

Keyword: Star-X-ray; Star-variable; Star-late-type; Star-HD 81032

\section{Introduction}

The presence of strong X-ray and nonthermal radio emission in late-type stars is a well-known indication of enhanced coronal activity (Drake et al. 1992; Güdel 2002). X-ray emission from coronae $\left(T_{e} \sim 10^{6-7} \mathrm{~K}\right.$ ) and chromospheric emission $\left(T_{e} \sim 10^{4} \mathrm{~K}\right)$ are also closely correlated, and thus stars with intense coronae will have strong chromospheric emission, as evidenced by their UV and Ca II H and K line emission (e.g. Ayres et al. 1995). While many active stars have been identified as such through their X-ray and radio emission, it is only through detailed optical photometric and spectroscopic studies that their activity can be classified into known types.

RS CVn binaries are perhaps the most common type of active star, at least for stars having spectral types in the range from late $\mathrm{F}$ through $\mathrm{K}$. In an RS CVn binary, the two stars are usually tidally locked so that the rotational period of each star is approximately the same as the orbital period; however, the two stars are not undergoing mass transfer (i.e. they form a detached system). One star is generally a spectral type $\mathrm{F}$ to $\mathrm{G}$ dwarf or a subgiant that is $\sim 1000 \mathrm{~K}$ hotter than its companion, which is usually a $\mathrm{G}$ to $\mathrm{K}$ type giant or sub-giant. In the more recent definition of the RS CVn class, there is no restriction on the spectral type of the secondary star or on the orbital period, except for the evolutionary constraint (Fekel et al. 1986). RS CVns have been further subdivided into three groups according to their orbital period: the short period $(P \leq 1 \mathrm{~d})$, classical $(1 \mathrm{~d} \leq \mathrm{P} \leq 14 \mathrm{~d})$, and long period ( $P \geq 14 \mathrm{~d})$ groups. In the soft $(0.1-2.0 \mathrm{keV})$ X-ray band, the luminosities of RS CVn binaries typically lie in the range of $10^{29}-10^{32} \operatorname{erg~s}^{-1}$ (Drake et al. 1989), compared to $10^{25.5}-10^{29.5} \mathrm{erg} \mathrm{s}^{-1}$ for normal late-type stars (Schmitt \& Liefke 2004). VLA and ATCA observations at $\mathrm{cm}$ wavelengths have shown that the radio luminosities of RS CVn systems are in the range from $10^{14.5}$ to $10^{17.5} \mathrm{erg} \mathrm{s}^{-1} \mathrm{~Hz}^{-1}$ (Drake \& Linsky

\footnotetext{
*E-mails:jeewan@aries.ernet.in; singh@tifr.res.in;drake@olegacy.gsfc.nasa.gov; sagar@aries.ernet.in
} 
1986, Drake et al.1989), which is also enhanced compared to that found for other late-type stars, typically $\leq 10^{15} \mathrm{erg} \mathrm{s}^{-1} \mathrm{~Hz}^{-1}$ (Güdel 2002).

The K0 IV star HD 81032 (Houk \& Smith-Moore 1988) was first noticed as an X-ray active star when it was identified as the likely optical counterpart of a soft X-ray (0.2-4.0 keV) source in the Einstein Slew Survey, 1ES 0920-13.6, with an X-ray flux of $2.1 \pm 0.9 \times 10^{-11} \mathrm{erg} \mathrm{cm}^{-2} \mathrm{~s}^{-1}$ (Elvis et al. 1992, Schachter et al. 1996). An X-ray source at this position was later detected in the ROSAT All-Sky-Survey (RASS) at a weaker (but statistically more significant) level of $3.9 \pm 0.4 \times 10^{-12} \mathrm{erg} \mathrm{cm}^{-2} \mathrm{~s}^{-1}$, named $1 \mathrm{RXS}$ J092253.7-134919 in the RASS Bright Source Catalogue (Voges et al. 1999). This star was detected on February 12, 1993 as a radio source, with a $3.6 \mathrm{~cm}$ flux of $0.68 \pm 0.05$ mJy (Drake, S. A., private communication). Given its spectral type and luminosity class, and its V-magnitude of 8.91 (Wright et al. 2003), the spectroscopic distance of HD 81032 is $140 \pm 45$ pc, implying an X-ray luminosity of $9.2 \pm 4.2 \times 10^{30} \mathrm{erg} \mathrm{s}^{-1}$ (based on the RASS count rate and an assumed conversion factor of $6 \times 10^{-12}$

erg $\mathrm{cm}^{-2}$ PSPC $\mathrm{cts}^{-1}$ : a self-consistent X-ray luminosity based on the RASS data is calculated in $\left.\S 8\right)$, and a radio luminosity of $1.6 \pm 0.7 \times 10^{16} \mathrm{erg} \mathrm{s}^{-1} \mathrm{~Hz}^{-1}$, where the distance uncertainty is the dominant contributor to the large errors in the luminosities. These values clearly show that the star HD 81032 has an active corona.

In this paper, we present extensive optical photometric and spectroscopic observations, as well as an analysis of archival X-ray data, of the star HD 81032. This is the first detailed optical photometric and spectroscopic study of this star, although a brief discussion of some of the optical photometry of this star was given in Pandey et al. (2002). Based on our long-term optical study, the star HD 81032 is shown to be a new member of the (long-period) RS CVn class.

The organization of this paper is as follows. The observations and data reduction are presented in $\S 2$. In $\S 3$, we analyse the data for the periodicity. In $\S 4$, we discuss the light curve and phase of minimum light. Chromospheric emission features are described in $\S 5$. In $\S 6$ and $\S 7$, the spectral type and IR excess of HD 81032 are discussed, while the X-ray spectra are discussed in $\S 8$. Finally, $\S 9$ summarizes the main results of this paper.

\section{Observations and data reduction}

\subsection{Optical Photometry}

HD 81032 was observed in the Johnson B, V and Cousins R filter for 122 nights during four observing runs - year 2000-2001 (19 nights), year 2001-2002 (54 nights), year 2002-2003 (24 nights) and year 2003-2004 (25 nights) - at the Aryabhatta Research Institute of Observational Sciences (ARIES). The observations were made with the 104-cm Sampurnanand telescope and using a $2 k \times 2 k$ CCD camera in years $2000-$ 2003 , and a $1 k \times 1 k$ CCD camera during the years $2003-2004$. A few CCD frames were taken in the B, V and R filters on every night, with exposure times ranging from 2 to 60 secs, depending upon the seeing conditions and the filter used. Several bias and twilight flat frames were also taken during each observing run. Bias subtraction, flat fielding and aperture photometry were performed using IRAF ${ }^{\dagger}$.

${ }^{\dagger}$ IRAF is distributed by National Optical Astronomy Observatories, USA 
For the star HD 81032 the comparison and check stars were TYC 547113451 and USNO-A2.0 075006845737, respectively. Differential photometry, in the sense of variable minus the comparison star, was done, since program, comparison and check stars were all in the same CCD frame. UBVRI observations of HD 81032 along with some of the Landolt (1992) standard region SA 98 were obtained on 22 February 2004 for photometric calibration. The average magnitudes of HD 81032 during the observing years 2003 - 2004 in the U, B, V, R and I filters were $10.242 \pm 0.007,9.635 \pm 0.005,8.614 \pm 0.005,8.401 \pm 0.003$, and $7.736 \pm 0.003$, respectively.

\subsection{Optical Spectroscopy}

Spectroscopic observations were carried out during 2003 January 20 to 24 at the Vainu Bappu Observatory, Kavalur with the OMR spectrograph fed by the 234-cm Vainu Bappu Telescope. The data were acquired with a $1024 \times 1024 \mathrm{CCD}$ camera of $24 \times 24 \mu \mathrm{m}$ square pixel size covering a range of $1200 \AA$ and having a dispersion of $1.25 \AA /$ pixel. The star was observed in the wavelength ranges of $3500-4700 \AA$ and 5700 $6900 \AA$ A. Four spectra of HD 81032 in the $\mathrm{H} \alpha$ region and three in the CaII H and K region were obtained. a signal-to-noise of ratio between 20 to 40 was achieved in these spectra. HD 71952, a K0IV type star, was also observed as a reference star for HD 81032.

The spectra were extracted using the standard reduction procedures in the IRAF packages (bias subtraction, flat fielding, extraction of the spectrum and wavelength calibration using arc lamps). The spectral resolution was determined by using emission lines of arc lamps taken on the same nights. A spectral resolution $(\delta \lambda)$ of $2.7 \AA$ at $6300 \AA$ and $3.7 \AA$ at $4000 \AA$ was achieved. All the spectra were normalized to the continuum and equivalent widths for the emission lines were computed using the IRAF task splot. The errors in the measurement of the equivalent widths of the emission lines were determined by measuring the equivalent widths of some moderate absorption lines for each spectra. We computed the standard deviation for the equivalent width of each feature, and finally determined the mean standard deviation to be $0.02 \AA$.

\section{$2.3 \quad$ X-ray Data}

The star HD 81032 was observed and detected by the ROSAT PSPC detector during the ROSAT AllSky-Survey (RASS) phase over a 2-day period from 1990 November 10 to 12 . The exposure time was $501 \mathrm{~s}$, and was accumulated in 26 separate short scans of this region of sky. The PSPC had an energy range from $0.1-2.4 \mathrm{keV}$ with a (low) spectral resolution $(\Delta E / E \approx 0.42$ at $1 \mathrm{keV})$. A full description of the X-ray telescope and detectors can be found in Trümper (1983) and in Pfeffermann et al.(1987). The ROSAT X-ray data for HD 81032 were obtained from the public archives, the relevant RASS dataset being rs932025n00. Source spectra for HD 81032 were accumulated from on-source counts obtained

from a circular region on the sky centered on the X-ray peak and having a radius of 3.85 arcmin. The background was accumulated from several neighboring regions at nearly the same offset from the source. 


\section{Photometric light curves and period analysis}

Photometric light curves corresponding to the four observing runs were taken. Figure 1 shows the V band differential light curves of the star HD 81032 at different epochs. We did not find any significant variations in the comparison star (see below). The yearly mean of the standard deviation $(\sigma)$ between the different measures of comparison and check stars in the B, V and R filters was found to be 0.011, 0.01 and 0.01 , respectively. Each light curve shown in Fig. 1 was analysed for periodicity. To find a period from unequally spaced data, we used the CLEAN algorithm (Roberts et al. 1987) in Starlink's PERIOD software. The power spectrum obtained using this method is shown in the inset of each panel of Fig. 1 along with the period determined. The CLEANed power spectra presented were obtained after 100 iterations with a loop gain of 0.1. The period is found to be constant within error for each epoch. To improve the period determination of the star HD 81032, the entire data from 2000 - 2004 were analysed using the same algorithm. Figure 2 shows the CLEANed power spectrum from the entire dataset. The highest peak in the CLEANed power spectrum corresponds to a period of $18.802 \pm 0.074 \mathrm{~d}$. The $18.802 \mathrm{~d}$ period is much more plausible than the $57 \mathrm{~d}$ period reported earlier (Pandey et al. 2002). The previous determination was mainly due to observational limitations as our early data were too sparse and highly uneven. Besides a subgiant like this one is unlikely to be synchronized in a binary of 57-days period.

\section{Photometric variation and phase of minima}

The Julian days of the observations were converted to the phases using the ephemeris:

$$
\operatorname{Phase}(\theta)=J D 2452307.761+18^{d} .802 E
$$

where the initial epoch corresponds to the conjunction with the first minimum observed. Figure 3 shows the differential $\mathrm{B}, \mathrm{V}, \mathrm{R}$ and $\mathrm{V}_{c}$, light curves and, (B-V) and (V-R) colour curves of the star HD 81032. Here $\mathrm{V}_{c}$ stands for the differential $\mathrm{V}$ band light curve of the comparison and the check stars. Each point in the light curves is mean of $3-4$ independent observations taken over a night. The light curve during the observing years 2001-2002 has a dense temporal coverage. We, therefore, divided this light curve into two different epochs to see any variation in the $\theta_{\min }$ and the amplitude. The mean epoch of the light curves, the observed maximum $\left(\Delta V_{\max }\right)$ and minimum $\left(\Delta V_{\min }\right)$ in the $\mathrm{V}$ band, peak to peak amplitude $\left(\Delta V=\Delta V_{\max }-\Delta V_{\min }\right)$, and phase of minima $\left(\theta_{\min }\right)$ are listed in Table 1 . The value of $\Delta V_{\max }$ was constant during each epoch indicating that the brightness of unspotted photosphere was constant from epoch to epoch. However, the value of $\Delta V_{\min }$ was reduced by 0.14 mag from epoch 'a' to epoch 'b', and remained constant during the epoch 'c', 'd' and 'e'.

The RS CVn systems usually show one or two well defined minima, thereby indicating that the rotational modulations caused by one or two prominent spots or groups of spots. Additional spots may be present at other longitudes, or in the circumpolar regions but contribution to the overall rotational modulation may not be appreciable. The phase of the light minimum $\left(\theta_{\min }\right)$ directly indicates the mean longitude of the dominant groups of spots. A sharp minimum was observed during the epoch 'a' (see Fig. 3a). At the same time the amplitude of the $\mathrm{V}$ band light curve was found to be 0.288 mag, which was maximum during our observations. The sharpness of the minimum indicate that it is the latitudinal 
extent of the groups of spots that may be responsible rather than the longitudinal extent. Broad minima during the epochs 'b', 'c' and 'd' indicate that the spots were spread over an appreciable longitudinal range (Fig. $3 \mathrm{~b}$ to d). It is interesting to see the light curve of the star HD 81032 during the epoch 'e'. Here a single large spot, characterized by a broad minimum during the epochs 'b', 'c' and 'd' separated into two groups of spots. This can be easily seen by two well separated minima (see Fig. 3 e). Significant change in $\theta_{\min }$ (see Table 1 ) is probably associated with a change in the spot configuration on the surface of the star.

Variation in the colour of the star HD 81032 is correlated with its magnitude (see top two panels of Fig. 3 a to e) i.e. the star becomes redder when fainter, and bluer when brighter, supporting the starspot hypothesis. The significance of the correlation has been calculated by determining the linear correlation coefficient, $r$, between the magnitude and the colours. The value of $r$ between $\mathrm{V}$ and $(\mathrm{B}-\mathrm{V}), \mathrm{V}$ and $(\mathrm{V}-\mathrm{R})$ and $(\mathrm{B}-\mathrm{V})$ and $(\mathrm{V}-\mathrm{R})$ was found to be $0.20,0.55$ and 0.40 , with the corresponding probability of no correlation being $0.0253,6.157 \times 10^{-12}$ and $2.443 \times 10^{-6}$, respectively. The conventional starspot model assumes that the spots are cooler than the surrounding photosphere, and hence one would expect the star to be the reddest at the light minimum, although flaring activity can lead an opposite effect being observed as seen in UX Ari (Ulvås \& Henry 2003; Padmakar \& Pandey 1999; Ravindran \& Mohin 1995).

Figure 4 shows the plot of the mean epoch versus phase minima $\left(\theta_{\min }\right)$ of the $\mathrm{V}$ band light curves. The following equation was fitted to the data by the method of linear least squares to determine the rate of the phase shift (or migration period).

$$
\theta_{\text {min }}=\omega\left(t-t_{0}\right)+\theta_{0},
$$

Here $\omega=2 \pi / P$ is the rate of phase shift (degrees/day), $\mathrm{P}$ is the corresponding period in days, $\mathrm{t}$ is time in days, $t_{0}$ is reference time, and $\theta_{0}$ is the reference spot longitude in degree. After the epoch JD 2452400 (or after the epoch 'd'), the evolution of different spots seems to be more responsible than the migration of single group of spots, so, we have used only first three points of the Fig. 4 (i.e. the phase minima of epoch 'a', 'b' and 'c') in the linear least square fit of equation (1). The solid line in Fig. 4 shows the linear least square fit of the equation (1) to the data. The rate of phase shift for the group of spots was determined as $0.1348 \pm 0.002$ degree/day, which corresponds to a migration period of $7.43 \pm 0.07$ years. The smaller group of spots during the epoch 'e' appears to rotate with the similar angular velocity with that of a group of spots during the epoch 'a', 'b' and 'c' (see the dotted line in Fig. 4). However, long-term studies of spot groups' evolution and migration in the star HD 81032 require much more frequent sampling in the time domain. The arrangement of groups of spots in one permanent strip can be easily interpreted as a long lived active group rotating with the same velocity. The open circles in the Fig. 4 are not following that strip, and may be due to the formation of a new group of spots on the surface of the star.

\section{$5 \quad \mathrm{H} \alpha$ and $\mathrm{CaII} \mathrm{H}$ and $\mathrm{K}$ emission lines}

The $\mathrm{H} \alpha$ and CaII $\mathrm{H} \& \mathrm{~K}$ emission lines are important indicators of chromospheric activity. In very active stars like II Peg, V711 Tau, UX Ari the $\mathrm{H} \alpha$ emission is seen clearly above the continuum, whereas in less 
active stars only a filled in absorption line is observed. Figure 5 shows spectra of HD 81032 in the H $\alpha$ region. Spectrum of the star HD 71952 is shown for comparison. $\mathrm{H} \alpha$ emission line can be seen clearly in the star HD 81032 (Fig. 5). It has been suggested that in the RS CVn systems at least one star must also show the intense emission in the $\mathrm{H}$ and $\mathrm{K}$ lines of CaII (Fekel et al. 1986). As shown in Figure 6, $\mathrm{CaII} \mathrm{H}$ and $\mathrm{K}$ lines are above the continuum at all the phases observed. The measured equivalent widths (EWs) of the $\mathrm{H} \alpha$, CaII H and $\mathrm{K}$ emission feature, the corresponding JD and phase are listed in Table 2.

The photometric observations during the epoch 'd' were close to the spectroscopic observations. Figure 7 shows the plot of EWs of $\mathrm{Ca}$ II $\mathrm{H}, \mathrm{K}$ and $\mathrm{H} \alpha$ against phase, along with the $\mathrm{V}$ band light curve of the epoch 'd'. It appears that $\mathrm{H} \alpha$, Ca II H, and $\mathrm{K}$ emission features are variable and anti-correlated with the photometric phase i.e. the maximum at photometric minimum and minimum at photometric maximum. This could be due to the presence of active cool spots on the surface of the star.

\section{Spectral type}

The value of the total galactic reddening $\mathrm{E}(\mathrm{B}-\mathrm{V})$ in the direction of $\mathrm{HD} 81032$ is estimated to be $0.04 \mathrm{mag}$ from the reddening map given by Schlegel et al. (1998). However, the star HD 81032 suffers negligible reddening at a distance of $140 \mathrm{pc}$, and we assume $\mathrm{E}(\mathrm{B}-\mathrm{V})=0$ for the remaining discussion. Using the Tycho parallax (Hog 1997) and the value of $V(=8.63 \mathrm{mag}$ ) mag from the present photometry the absolute magnitude $M_{V}$ of HD 81032 is 3.04 mag. The value of $M_{V}$ is consistent with the luminosity class IV for this star. The $(B-V)$ colour of HD 81032 derived from our photometry is $1.02 \pm 0.01$ consistent with the relatively precision to the value of $0.98 \pm 0.05$ given in the Tycho catalogue (Wright et al. 2003). The (B-V) colour is best matched with the spectral class K0IV, and is consistent with the spectral class identified by Wright et al. (2003). Applying the bolometric correction -0.4 (Schmidt \& Kaller 1982) for K0IV star, the bolometric magnitude of HD 81032 is 2.65 mag.

\section{Spectral energy distribution (SED)}

We have determined the SED of HD 81032 using broad band UBVRI (present photometry), 2MASS JHK (Cutri et al. 2003) and 12, 25, 60 and $100 \mu$ IRAS (Moshir 1989) fluxes. However, only upper limits are available at 25,60 and $100 \mu \mathrm{m}$. The observed SED of HD 81032 along with the synthetic SED is shown in Figure 8. The synthetic SED is expected from the intrinsic properties of the star (Kurucz 1993). The model SED shown in Fig. 8 has been adjusted to coincide with the observed SED of the star HD 81032 at the V-band wavelength of $0.55 \mu$. We have overplotted the synthetic SED for the different $T_{\text {eff }}$ and $\log$ combinations. The values of $T_{\text {eff }}$ and logg which best match the observed SED are $5000 \pm 250 \mathrm{~K}$ and $3.5 \pm 0.5$, respectively, and are consistent within one subclass of the inferred K0IV spectral type of the star HD 81032 (see $\S 6$ ).

The continuum of HD 81032 is in good agreement with the normal values for the spectral type of K0IV up to JHK band but deviates in all bands longer than $25 \mu m$ (see Fig. 8). The difference between the observed and the expected $(\mathrm{J}-\mathrm{K})$ and $(\mathrm{H}-\mathrm{K})$ colours are $0.06 \pm 0.04$ and $0.005 \pm 0.03 \mathrm{mag}$, respectively. This indicates that there is no significant colour excess in $\mathrm{J}, \mathrm{H}$ and $\mathrm{K}$ band. The expected $(\mathrm{J}-\mathrm{K})$ and 
(H-K) colours were taken from the spectral type of the star (Koorneef, 1983). The $12 \mu \mathrm{m}$ magnitude $\left(m_{12}\right)$ of the star HD 81032 was found to be $6.09 \pm 0.17 \mathrm{mag}$ using the relation $m_{12}=-2.5 \log f_{12}+3.63$ (Mitrou et al. 1996). The intrinsic [K-12] colour of the star HD 81032 is determined to be $0.03 \pm 0.17$ mag. The expected $[K-12]$ colour of 0.14 mag for the K0IV type star (Verma et al. 1987) gives the colour excess of $0.17 \mathrm{mag}$, which is consistent with the ideal value of $0.0 \pm 0.2 \mathrm{mag}$ (Mitrou et al. 1996).

\section{$8 \quad$ X-ray light curve and spectra}

The light curve for the source and background were extracted using the xselect package for the PSPC 0.1 - $2.4 \mathrm{keV}$ energy band which contains all the X-ray photons. The background-subtracted X-ray light curve of HD 81032 is shown in Figure 9 plotted with a time bin size of $64 \mathrm{~s}$. It appears from the light curve that a moderate flare occurred during the RASS observations, with a peak of about $0.6 \mathrm{ct} \mathrm{s}^{-1}$ (compared to a pre-flare level of $\left.0.2-0.3 \mathrm{ct} \mathrm{s}^{-1}\right)$ at approximately JD $=2448206.95(1990 / 11 / 11$ 10:48:0.0) and a half-decay time of $2.6 \times 10^{4} \mathrm{~s}$.

The X-ray spectrum of HD 81032 as observed with the ROSAT PSPC is shown in Figure 10. Response matrices based on the available off-axis calibration of the PSPC and using the appropriate ancillary response files were created and data were fitted using the xspec (version 11.3.1) spectral analysis package. Spectral models for thermal equilibrium plasma known as the Mewe-Kaastra-Liedahl or MEKAL model (Liedahl, Ostrecheld \& Goldstein 1995; Mewe, Kaastra \& Liedahl 1995) were used. The backgroundsubtracted X-ray spectra were fitted with 1-temperature (1T) and 2-temperature (2T) plasma models, either assuming solar photospheric abundances as given by Anders \& Grevess (1989) or allowing the abundance of every element other than $\mathrm{H}$ to vary by a common factor relative to the solar (photospheric) values. In each of the above models the interstellar absorption was assumed to follow the absorption crosssections given by Morrison \& McCammon (1983), and the total intervening hydrogen column density $N_{H}$ was allowed to vary freely.

The results of different model fits are summarized in Table 3. Single-temperature MEKAL models with abundances fixed to the solar values gave unacceptably high values for $\chi_{\nu}^{2}$, and thus can be rejected. However, single-temperature plasma models with abundances of $0.19_{-0.08}^{+0.14}$ times solar and plasma temperature of $0.84_{-0.20}^{+0.17}$ were found acceptable. Alternatively, two-temperature MEKAL models with fixed solar abundances were also found to be acceptable; the two temperatures being $0.2_{-0.1}^{+0.2} \mathrm{keV}$ and $1.12_{-0.36}^{+0.52} \mathrm{keV}$. Multi-temperature variable abundance models would also produce acceptable fits to the PSPC spectrum, but the small number of accumulated counts does not warrant such complex models. The PSPC spectrum and the best-fit two-temperature plasma model with solar abundances are shown in Fig. 10 along with the significance of the residuals in terms of their $\chi^{2}$.

Based on the best fit 2T MEKAL model a source flux of $9.1_{-1.6}^{+0.9} \times 10^{-12} \mathrm{erg} \mathrm{cm}^{-2} \mathrm{~s}^{-1}$ was obtained. At a distance of $140 \mathrm{pc}$ the X-ray luminosity of HD 81032 is calculated to be $2.1_{-0.4}^{+0.2} \times 10^{31} \mathrm{ergs}^{-1}$, similar to that of known subgiants RS CVn binaries (Drake et al. 1989, Singh et al. 1995,1996; Dempsey et al. 1997; Padmakar et al. 2000; Pandey et al. 2005). The derived X-ray luminosity is about a factor of 2 bigger than the earlier estimate given in $\S 1$. This may be due to the non-trivial column density of 
$1.4 \times 10^{20} \mathrm{~cm}^{-2}$ inferred: objects with a high column density will typically have a higher count rate to flux conversion factor than the standard one which assumes a low column density. The values of $k T_{1}$ and $k T_{2}$ of HD 81032 (see Table 3) are consistent with those of other RS CVn systems studied by Dempsey et al. (1997), who found an average value of $0.18 \pm 0.01 \mathrm{keV}$ and $1.37 \pm 0.04 \mathrm{keV}$ for a sample of $28 \mathrm{RS}$ CVn systems. The volume emission measures EM1 and EM2 of HD 81032 are also found to be consistent with those of the other similar systems, where the average volume emission measures $E M_{1}$ and $E M_{2}$ are $1.2 \pm 0.3 \times 10^{53}$ and $5.7 \pm 1.2 \times 10^{53} \mathrm{~cm}^{-3}$, respectively (Dempsey et al. 1997).

All the errors quoted in this section are internal statistical errors, and that the distance-dependent errors of $45 \%$ in luminosity which were mentioned in $\S 1$ have not been included in, for example, the EM errors quoted in Table 3 or the errors in the X-ray luminosity given the above paragraph of this section.

\section{Summary}

A photometric period of $18.802 \pm 0.074 \mathrm{~d}$ has been discovered in the star HD 81032 . The amplitude and the phase of the photometric light curves of HD 81032 are observed to be changing from one epoch to another. The amplitude of variation is found to be maximum (0.29 mag) during the observing year 2000-2001. The change in the amplitude is mainly due to a change in the minimum of the light curve, and this may be due to a change in the spot coverage on the surface of the star. A single group of spots is found to migrate, and a migration period of $7.43 \pm 0.07$ years is determined from the first three years of the data. The star is seen to be redder at the light minimum and we interpret this is due to the relatively cooler temperature of the darker regions present in the visible hemisphere. A spectral type of K0IV is determined for HD 81032.

The equivalent widths of $\mathrm{H} \alpha$, Ca II H and K emission lines in the spectra of HD 81032 appear more intense close to the photometric light minimum. This could be attributed to the presence of cool spots on the surface of the stars. The observed X-ray spectrum and the inferred coronal plasma parameters for HD 81032 are typical of those seen in active stars such as RS CVn binaries.

All of the optical and X-ray properties found for HD 81032 are most consistent with it being an evolved RS CVn binary of the long-period type. Although there are a handful of apparently single evolved stars, e.g., FK Com, which can exhibit similar properties, identification of HD 81032 as such a rare object seems rather improbable. Clearly, high-resolution optical spectroscopy of HD 81032 in order to confirm the presence of radial velocity variability and determine a spectroscopic binary orbit would be definitive evidence for its proposed binary nature.

\section{Acknowledgment}

We are grateful to the anonymous referee for valuable comments and suggestions. We are thankful to the time allocation committee for giving time at 234-cm VBT. This research has made use of data obtained from the High Energy Astrophysics Science Archive Research Center (HEASARC), provided by NASA's Godard Space Flight center. Starlink is funded by PPARC and based at the Rutherford Appleton Laboratory, which is part of Council for the Central Laboratory of the Research Councils, UK. 
This publication makes use of data products from the Two Micron All Sky Survey, which is a joint project of the University of Massachusetts and the Infrared Processing and Analysis Center/California Institute of Technology, funded by the National Aeronautics and Space Administration and the National Science Foundation.

\section{References}

[1] Anders, E., \& Grevesse, N. 1989, Geochim. Cosmochim. Acta, 53, 197.

[2] Ayres, T. R., et al., 1995, ApJS, 96, 223

[3] Cutri, R. M., et al., 2001, 2MASS All-Sky Catalog of Point Sources, VizieR On-line Data Catalog $\mathrm{II} / 246$

[4] Dempsey, R. C., Linsky, J. L., Fleming, T. A., \& Schmitt, J. H. M. M., 1997, AJ, 478, 358

[5] Drake, S. A. private communication

[6] Drake, S. A., Simon, J., \& Linsky, J. L., 1992, ApJS, 82, 31

[7] Drake, S. A., Simon, J., \& Linsky, J. L., 1989, ApJS, 71, 905

[8] Drake, S. A., \& Linsky, J. L., 1986, AJ, 91, 1229

[9] Elvis, E., Plummer, D., Schachter, J. \& Fabbiano, G., 1992 ApJs, 80, 257

[10] Fekel, F. C., Moffet, T. J., \& Hennry G. W., 1986, ApJs, 60, 551.

[11] Güdel, M., 2002, ARAA, 40, 217.

[12] Hog, E., et al., 1997, A\&A 323, L57.

[13] Houk, N. \& Smith-Moore, M. 1988, Michigan Catalogue of Two-Dimensional Spectral Types for the HD Stars, Vol. 4: -26 to -12 Degrees.

[14] Koorneef, J., 1983, A\&A, 128, 84.

[15] Kurucz, R. L., 1993, private communication

[16] Landolt, A. U., 1992, AJ, 104, 340

[17] Liedahl, D. A., Osterheld, A. L., \& Goldstein, W. H., 1995, ApJ, 438, L115

[18] Mewe, R., Kaastra, J. S., \& Liedahl, D. A., 1995, Legacy, 6, 16

[19] Mitrou, C. K., Doyle, J. G., Mathioudakis, M. \& Antonopolou, 1996, A\&AS, 115, 61.

[20] Morrison, R., \& McCammon, D., 1983, ApJ, 270, 119

[21] Moshir, M., 1989, Explanatory Supplement to IRAS Faint Source Survey 
[22] Padmakar, P. S., Singh, K. P., Drake, S. A., \& Pandey, S. K., 2000, MNRAS, 314, 733

[23] Padmakar, P. S., \& Pandey, S. K., 1999, A\&AS, 138, 203.

[24] Pandey, J. C., Singh, K. P., Drake, S. A. \& Sagar, R., 2005, AJ, 130, 1231

[25] Pandey, J. C., Singh, K. P., Sagar, R. \& Drake, S. A., 2002, JApA, 23, 9.

[26] Pfeffermann, E., et al., in Soft X-ray Optics and Technology, Proc. SPIE, 733,519

[27] Raveendran, A. V., \& Mohin, S., 1995, A\&A, 301, 788.

[28] Roberts, D. H., Lehar, J. \& Dreher, J. W., 1987, AJ, 93, 968 p.493

[29] Schachter, J. F., et al. 1996, ApJ., 463, 747

[30] Schlegel, D. J., Finkbeiner, D. P., \& Davis, M., 1998, ApJ, 500, 525

[31] Schmidt - Kaler Th., 1982, In: Landolt-Bornstein, Numerical Data and Functional Relationship in Science and Technology, New series, Group VI, Vol. 2b, Scaifers K. \& Voigt H. H. (eds.) Springer Verlog, Berlin

[32] Schmitt, J. H. M. M. \& Liefke, C. 2004, A\&A, 417, 651.

[33] Singh, K. P., Drake, S. A., \& White, N. E., 1995, ApJ, 445, 840

[34] Singh, K. P., Drake, S. A., White, N. E. \& Simon, T., 1995, AJ, 112, 221

[35] Trümper, R. A. 1983, Adv. Space Res, 2, 241.

[36] Ulvås V. Aarum \& Henry, G. W., 2003, A\&A, 402, 1033.

[37] Verma, R. P., Iyenger, K. V. K., \& Rengarajan, T. N., 1987, A\&A, 177, 346

[38] Voges, W. et al., 1999, A\&A, 349, 389.

[39] Wright, C. O., Egan, M. P., Kraemer, K. E.,\& Price, S. D., 2003, AJ 125, 359. A\&A, 172, 225. 
Table 1: Parameters determined from the light curves of the star HD 81032.

\begin{tabular}{lccccc}
\hline $\begin{array}{l}\text { Mean epoch } \\
245000.0+\end{array}$ & Amplitude & $\Delta V_{\min }$ & $\Delta V_{\max }$ & \multicolumn{2}{c}{ phase minima } \\
\cline { 5 - 6 } (a) 1946.3350 & 0.288 & -2.739 & -3.027 & 0.01 & \\
& & & & & II \\
(b) 2245.8599 & 0.160 & -2.893 & -3.053 & 0.12 & \\
(c) 2322.6527 & 0.156 & -2.896 & -3.052 & 0.15 & \\
(d) 2621.4426 & 0.152 & -2.890 & -3.042 & 0.07 & \\
(e) 2983.8913 & 0.175 & -2.891 & -3.066 & 0.89 & 0.40 \\
\hline
\end{tabular}

Table 2: Equivalent widths (EWs) of $C a I I H \& K$ and $\mathrm{H} \alpha$ emission lines

\begin{tabular}{lcccc}
\hline JD & Phase & \multicolumn{3}{c}{ EWs $(A)$} \\
\cline { 3 - 5 } $245000+$ & & CaIIK & $H$ & $\mathrm{H} \alpha$ \\
\hline 2659.626 & 0.71 & 1.828 & 1.037 & - \\
2660.513 & 0.76 & - & - & 0.980 \\
2661.520 & 0.82 & 4.393 & 2.661 & 1.178 \\
2662.518 & 0.87 & 5.012 & 2.949 & 1.651 \\
2663.536 & 0.92 & - & - & 1.680 \\
\hline
\end{tabular}

Table 3: Results of X-ray spectral analysis

\begin{tabular}{|c|c|c|c|c|c|c|c|c|}
\hline Model & Abundances $^{a}$ & $\begin{array}{c}N_{H} \\
10^{20} \mathrm{~cm}^{-2}\end{array}$ & $\begin{array}{c}k T_{1} \\
(\mathrm{keV})\end{array}$ & $\begin{array}{c}E M_{1} \\
10^{52} \mathrm{~cm}^{-3}\end{array}$ & $\begin{array}{c}k T_{2} \\
(\mathrm{keV})\end{array}$ & $\begin{array}{c}E M_{2} \\
10^{53} \mathrm{~cm}^{-3}\end{array}$ & $\chi_{\nu}^{2}$ & $\begin{array}{l}\text { Degrees of } \\
\text { freedom }\end{array}$ \\
\hline MEKAL $1 \mathrm{~T}$ & 1.0 (fixed) & $0.21_{-0.16}^{0.22}$ & $0.85_{-0.10}^{+0.29}$ & $5.2_{-2.5}^{+2.2}$ & & & 1.87 & 17 \\
\hline MEKAL $1 \mathrm{~T}$ & $0.19_{-0.08}^{0.14}$ & $1.5_{-1.0}^{+1.0}$ & $0.84_{-0.17}^{+0.20}$ & $18.9_{-9.9}^{+14.7}$ & & & 1.53 & 16 \\
\hline MEKAL $2 \mathrm{~T}$ & 1.0 (fixed) & $1.4_{-1.1}^{+5.4}$ & $0.2_{-0.1}^{+0.2}$ & $2.9_{-1.5}^{+5.7}$ & $1.12_{-0.36}^{+0.52}$ & $1.8_{-1.6}^{+1.1}$ & 1.4 & 15 \\
\hline
\end{tabular}

${ }^{a}$ Common value of abundances for all the elements respect to the solar photosphere values; Errors are with $90 \%$ confidence for single parameter based on $\chi_{\min }^{2}+2.71$; distance $=140 \mathrm{pc}$. 

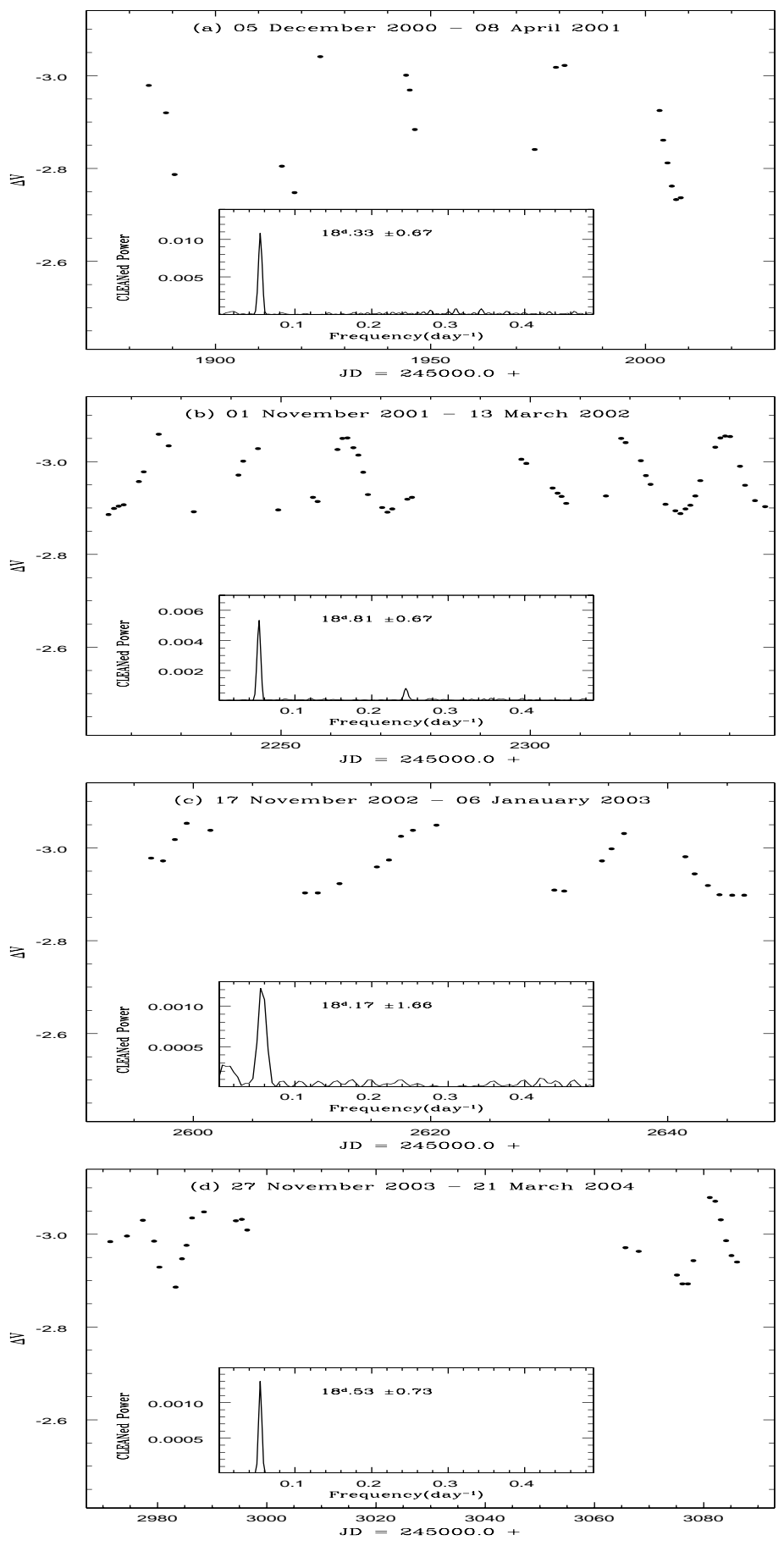

Figure 1: The V band light curves and corresponding CLEANed power density spectra (insets). The epoch is given at the top of each panel, and the period is written at the top of each inset. 


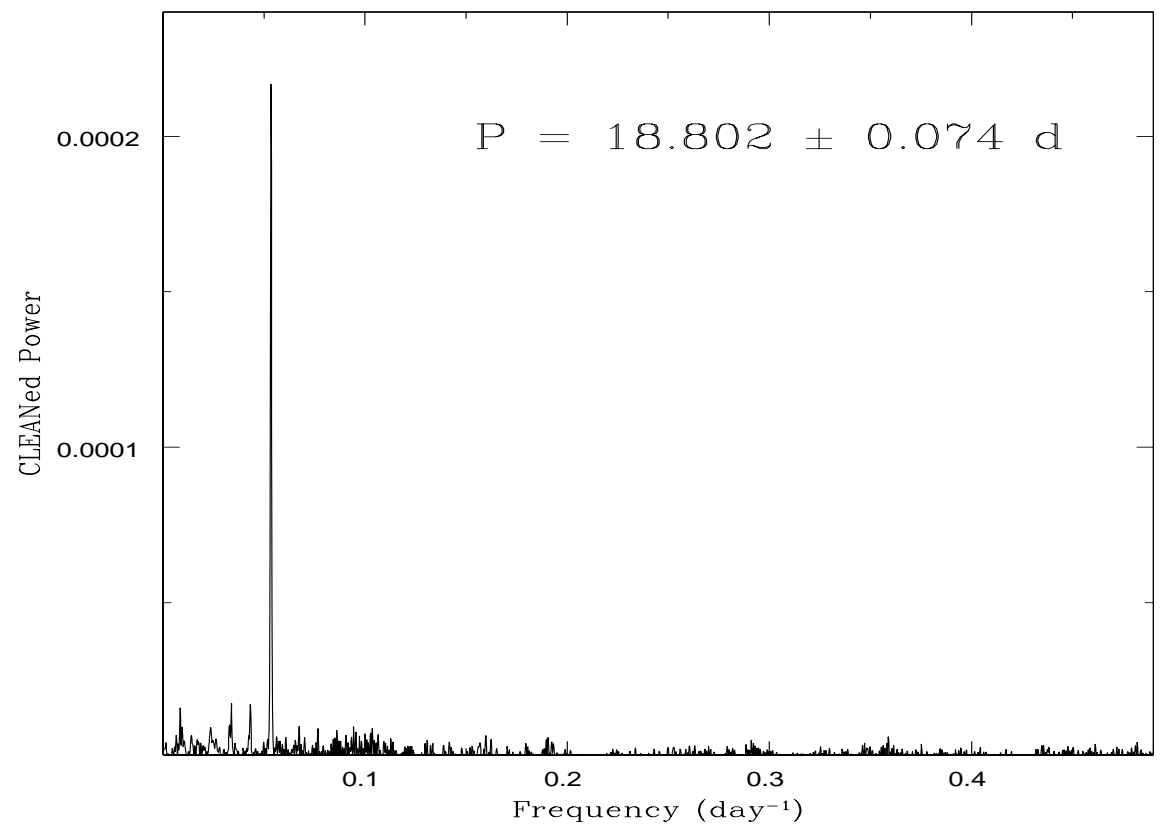

Figure 2: CLEANEd power spectra of whole data taken during 2000 - 2004 of HD 81032. 
(a) 1956.9950

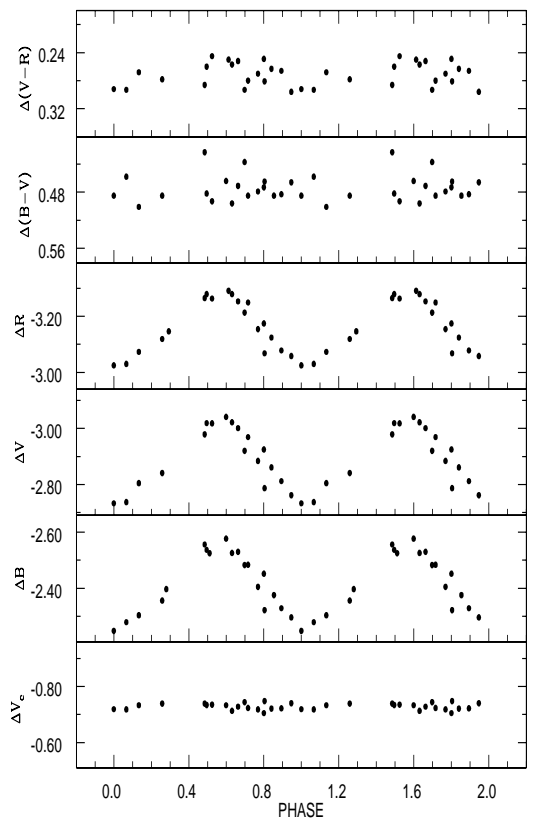

(c) 2325.610

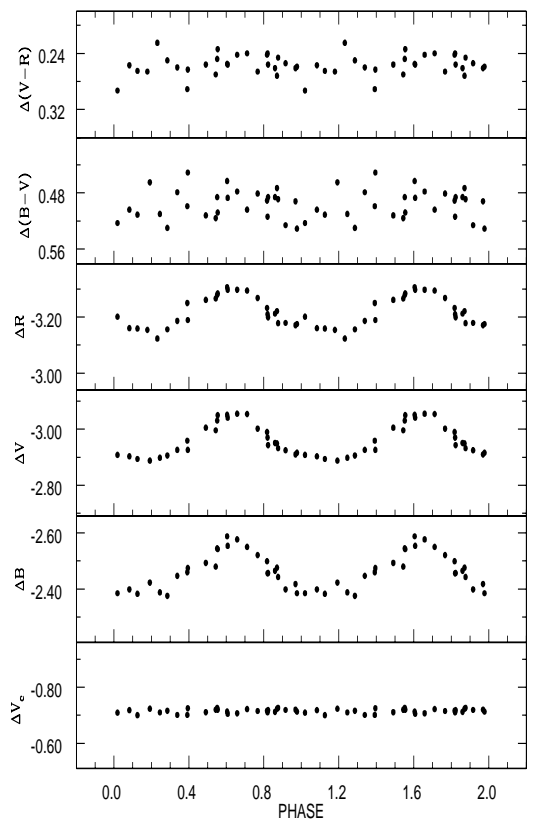

(b) 2248.500

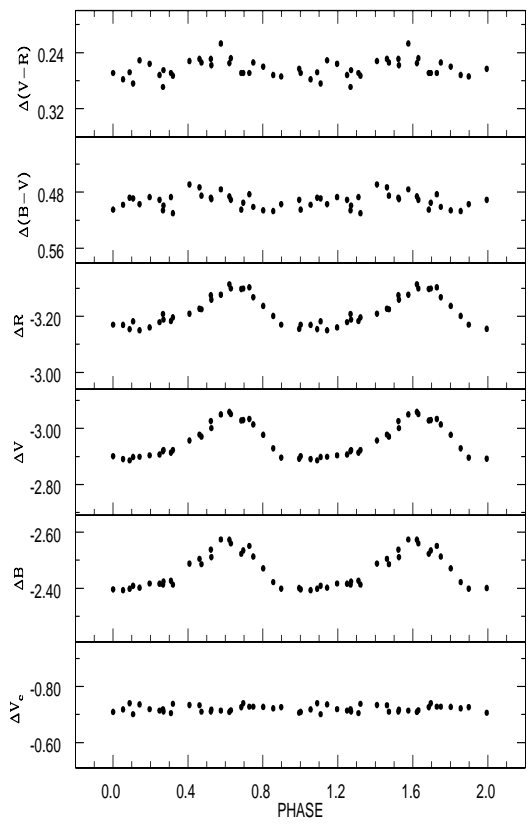

(d) 2622.710

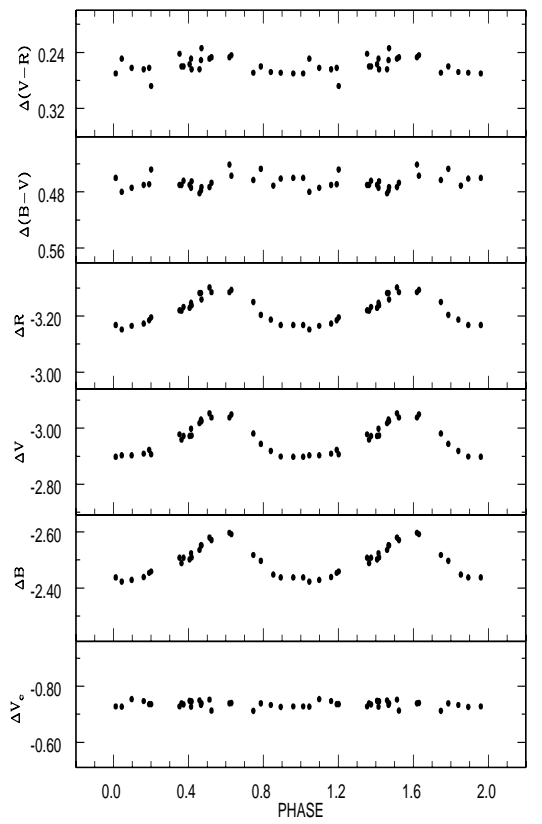

(e) 3029.566

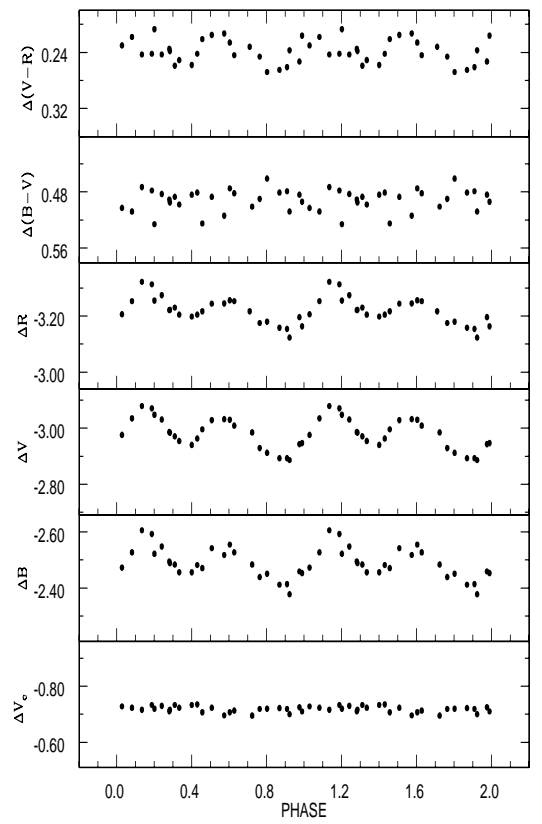

Figure 3: Differential $V_{c}, B, V, R$ light curve and $(B-V),(V-R)$ colour curve of HD 81032. $\Delta V_{c}$ stands for the differential magnitude between comparison and check stars. The light curve is folded using the period $18.802 \mathrm{~d}$. The epoch of each light curve is mentioned at the top of each figure. 


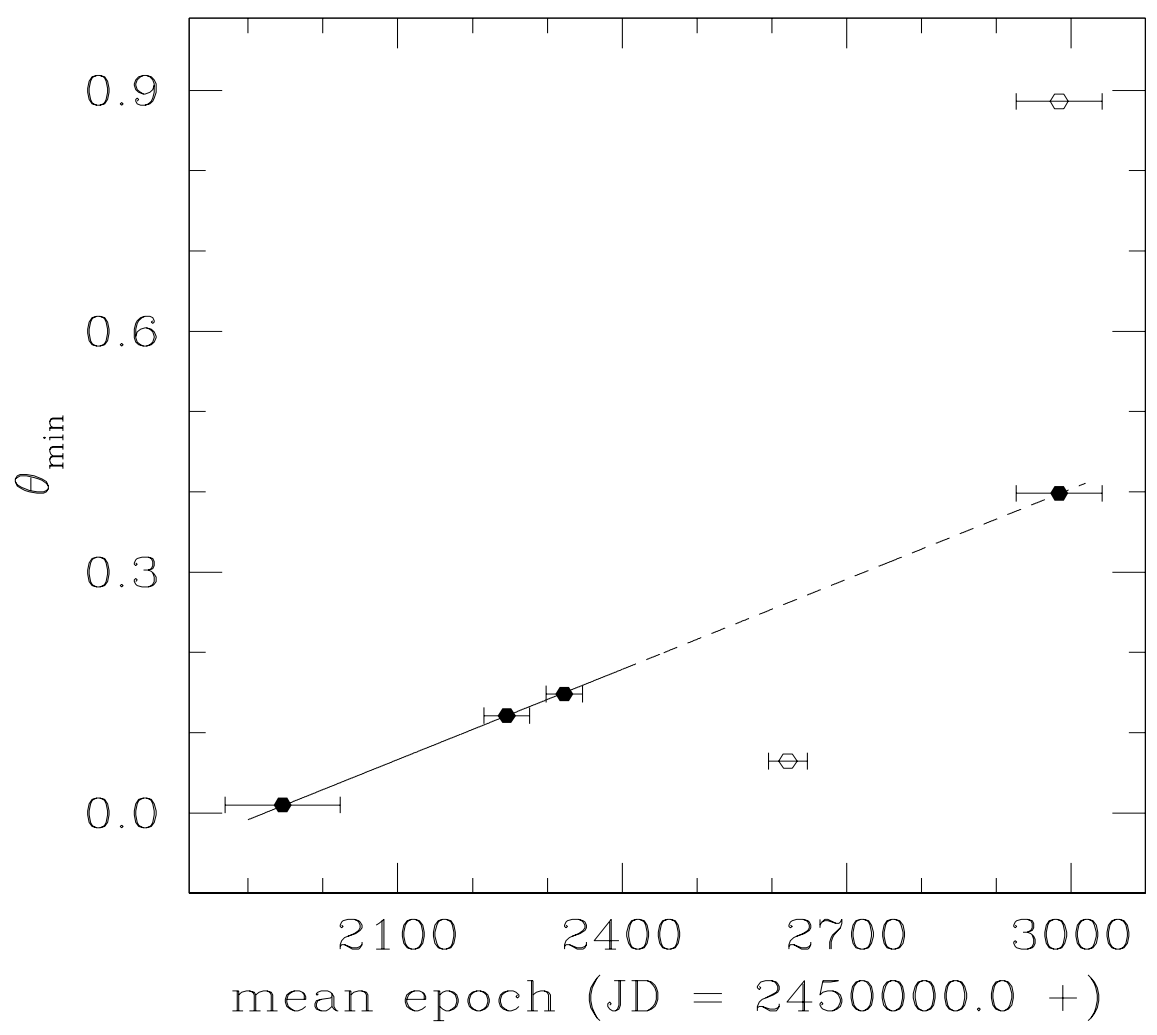

Figure 4: Plot of mean epoch versus light minima. The solid line represents the linear least square fit of the eq. 1 . The open circles were not used in the fit. The horizontal bar represent the length of the epoch. 


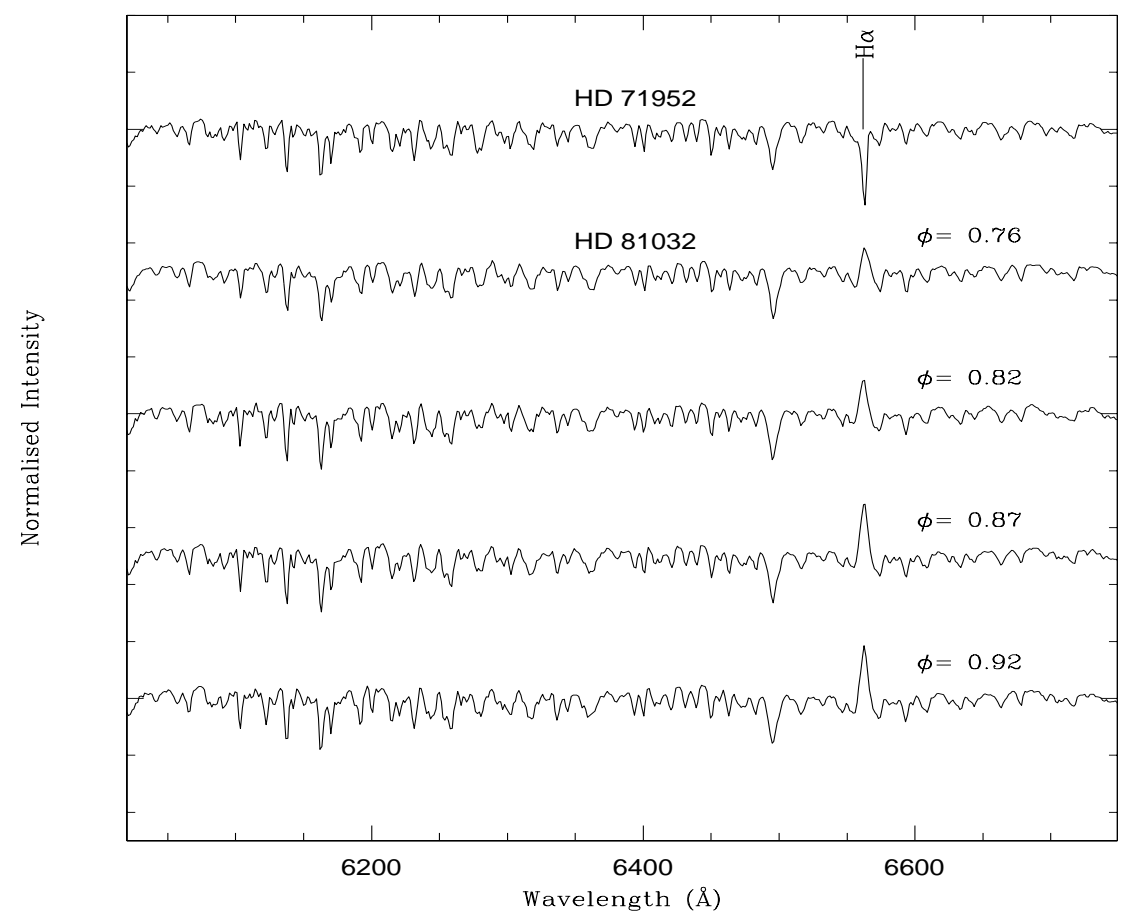

Figure 5: H $\alpha$ spectra of HD 81032. HD 71952 was taken as reference spectra.

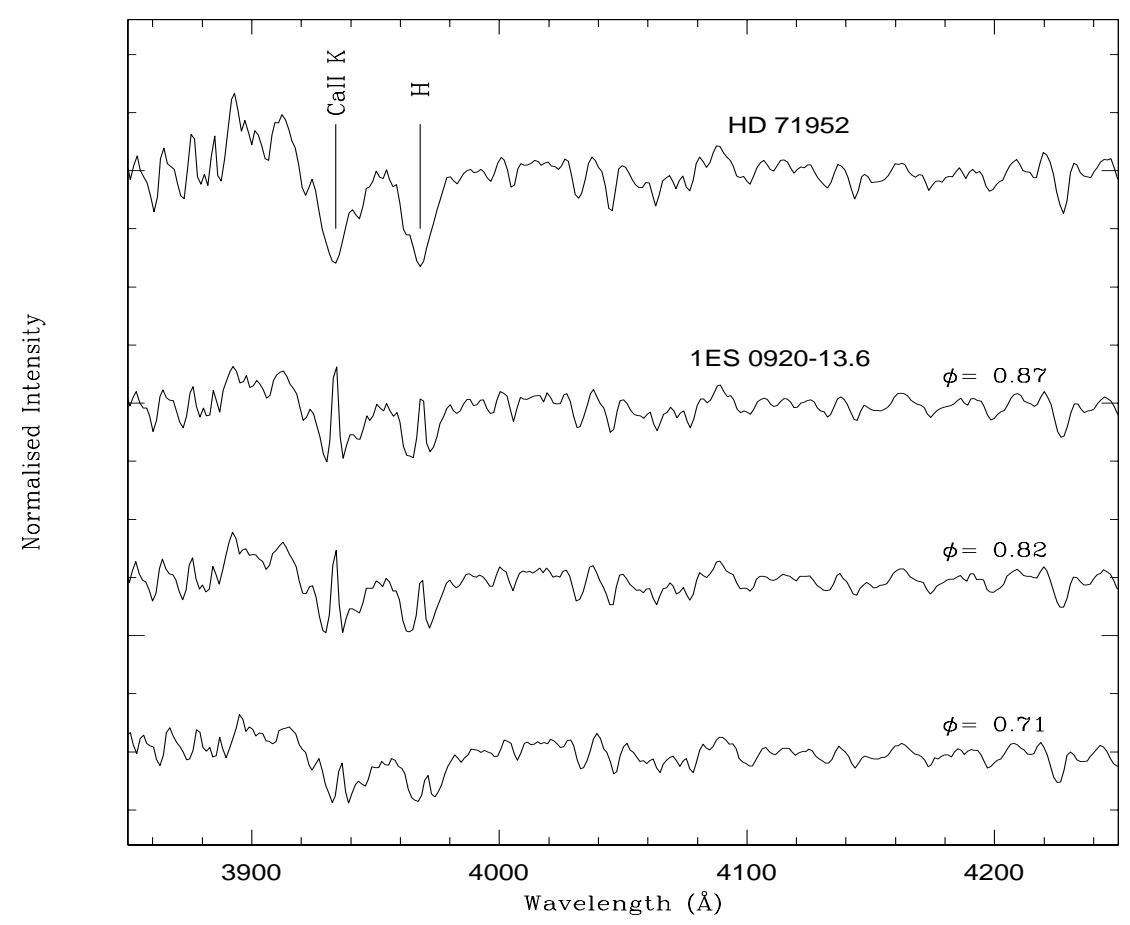

Figure 6: CaIIH\&K spectra of HD 81032. Spectra of HD 71952 was taken as a reference. 


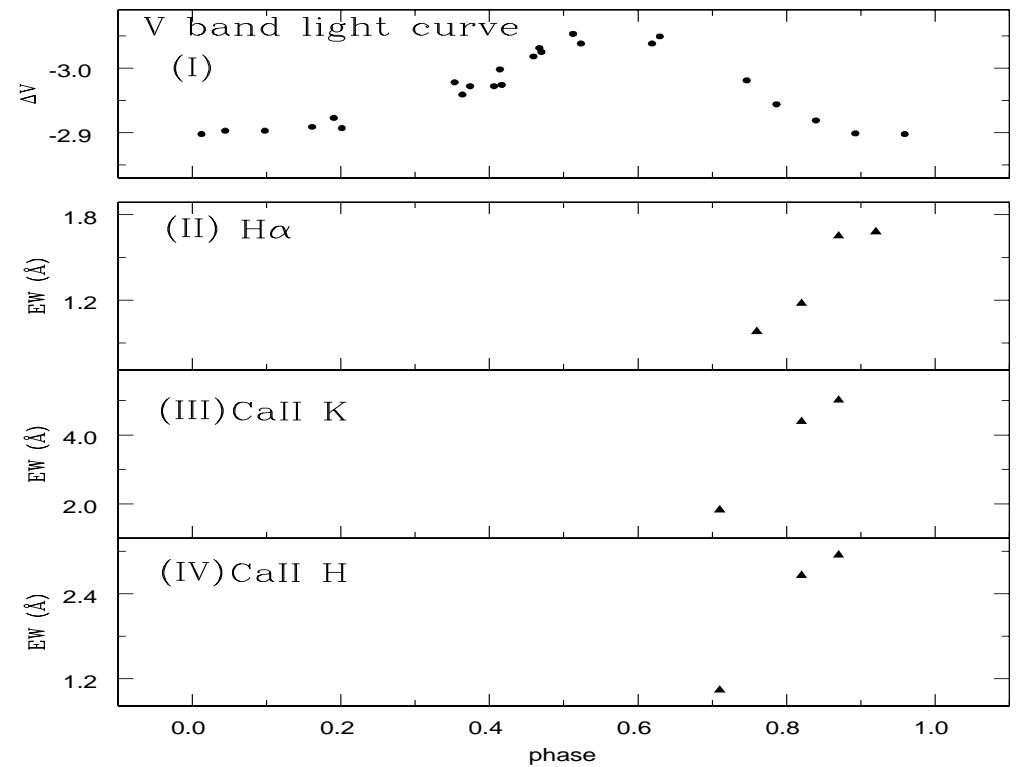

Figure 7: (I) V light curve of the HD 81032 during the epoch 'd', (II) variation of EWs of H $\alpha$ emission feature, (III) CaII K EWs and (IV) Ca II H EWs. Phases are reckoned from JD 2452307.761 and the period $18^{d} .802$.

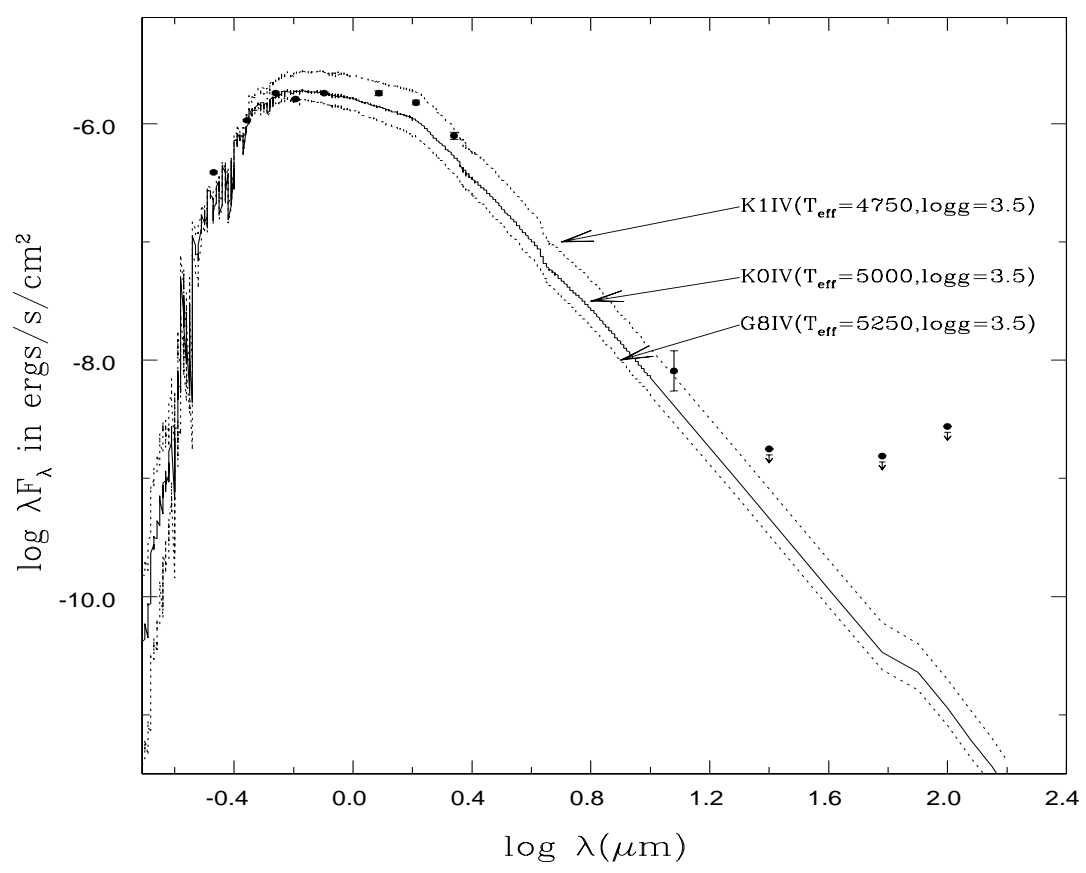

Figure 8: SED of the star HD 81032 (solid dots). The solid line represents the best match model SED from Kurucz (1993) as expected from the intrinsic properties of the star, dotted lines represent the SED of the K1IV and G8IV type star. 


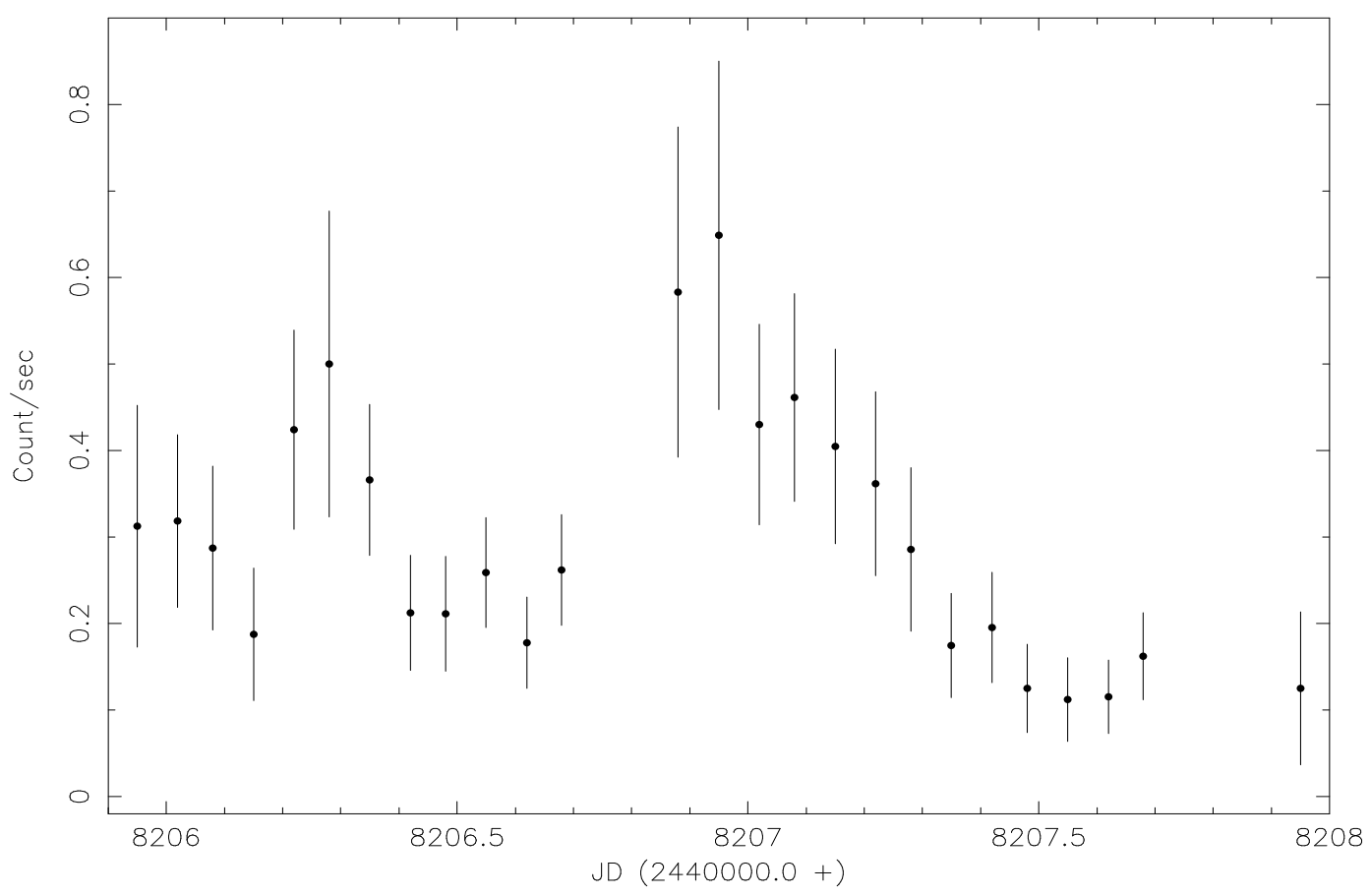

Figure 9: Background subtracted RASS PSPC X-ray light curve of HD 81032. The bin size in the light curve is $64 \mathrm{~s}$.

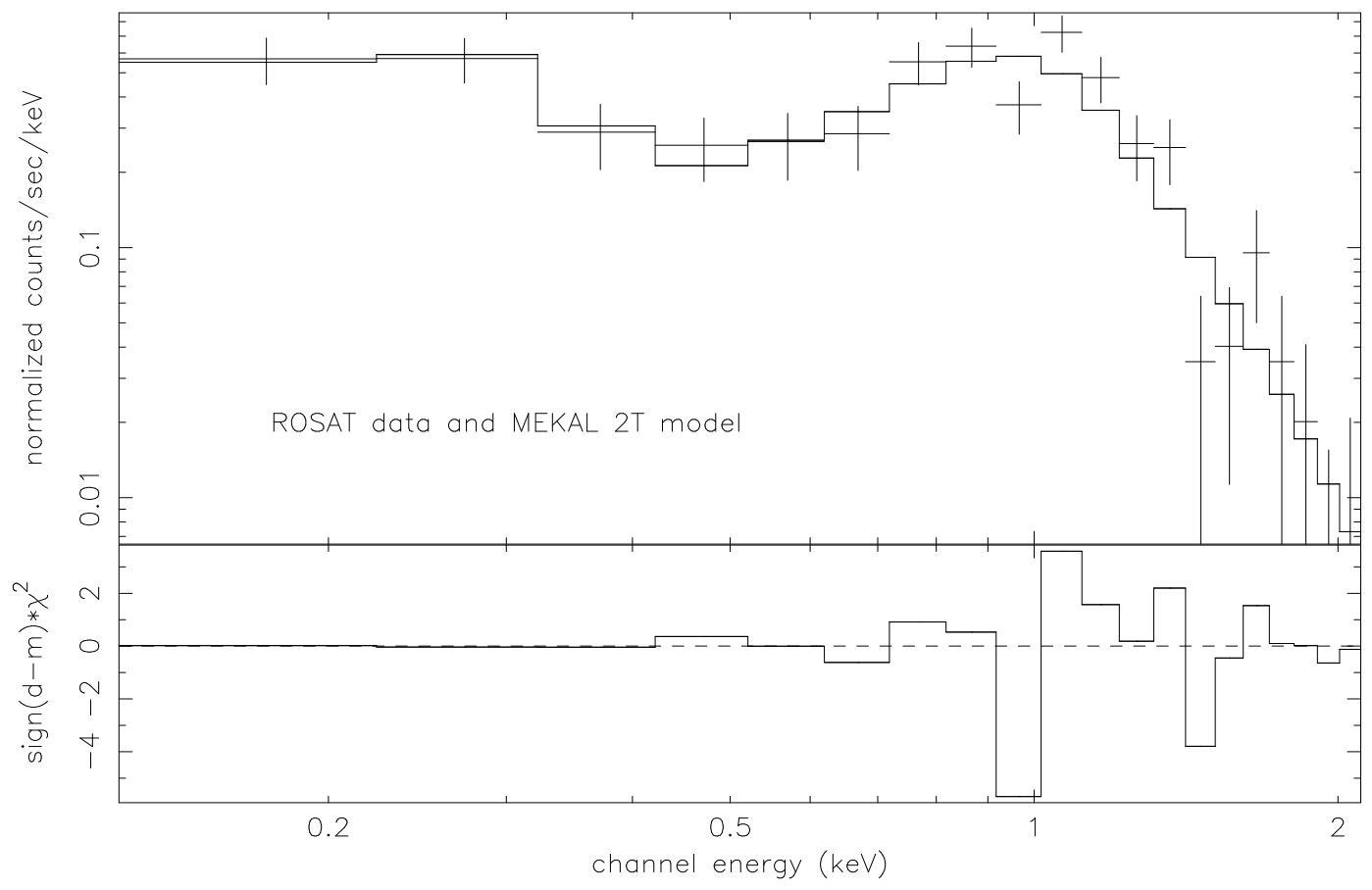

Figure 10: Spectra of HD 81032 taken with the ROSAT PSPC detector (top panel), the bottom panel shows the $\chi^{2}$ for each bin. 